\title{
Wireless data collection systems in the real world
}

\author{
PC Scott itmsoil Australia, Australia \\ J Paretas-Martinez Worldsensing, Spain \\ J Pérez-Arcas Worldsensing, Spain \\ KR Malcolm itmsoil Australia, Australia
}

\begin{abstract}
In the past, the instrumentation and monitoring component of any project was carried out manually. With the introduction of dataloggers, the transformation of how the data was collected, delivered and presented improved a quantum leap with near real time readings becoming possible and alarms being triggered. But in so doing, it did present an issue with massive cable runs being required to link all sensors to the system. These took a long time to install and were always difficult for the contractor to avoid damaging.

This paper will present the next generation of data collection through two different wireless systems each with their own unique capabilities. Firstly the Rippa wireless system where case studies in the Perth City Rail Link Project as well as the Gateway Project will be presented. Secondly, the Loadsensing mesh networked wireless system will be presented which has been successfully utilised in the Perth City Bus and Roy Hill Projects.
\end{abstract}

The details of each system will be examined in detail along with the applications and case studies.

In conclusion, the future of the wireless data collection systems will be discussed.

\section{Introduction}

Since Terzaghi, the observational technique of construction has been the best way to confirm that the predicted parameters of construction are being maintained. To enable this to be conducted in a more efficient manner the engineer has desired to have realtime data presented to their desktop computer in a timely and efficient manner. As technology improves the capability of instrumentation specialists to better provide this service has continued to be a work in progress. The development of dataloggers in the late 20th century saw major breakthrough in the capacity to achieve the goal. With telephone modem the data was presented with ease and in real time but on the construction site a problem developed in how to handle and protect the vast quantity of cable that was required to link all the sensors to a central datalogger.

Determining the best data delivery system for your instrumentation and monitoring program will have a large effect on the quality and reliability of the data, as well as initial and ongoing costs to your project. In selecting the most suitable data collection method, Sections 1.1 to 1.4 are taken into account.

\subsection{Purpose of the data}

If the monitoring is serving to form a baseline against which to compare behaviours during construction, then regular recordings will be required, but collection on a monthly or less basis is sufficient. Measurements used to control the construction process are often reviewed daily so daily delivery is sufficient. For monitoring critical structures or providing an alert function, much shorter intervals are required. 


\section{$1.2 \quad$ Type and number of sensors}

Nested or profile sensors will need a multichannel datalogger to monitor them. Some systems not originally designed for geotechnical instrumentation will not be compatible with vibrating wire (VW) sensors. Likewise multiplexed or serial sensors will require specific controllers in their respective dataloggers.

\subsection{Site layout and topography}

Distribution of monitoring locations, structures and landscape as well as cellular network availability will determine viable telemetry options within your site. Suitable cable routing options may be restricted, dictating the position and number of dataloggers required. Line of sight between monitoring locations will determine necessity for repeaters or additional gateways. Public access may mean the logger will need to be positioned in a safe positon away from the risk of vandalism. Hillocks or vegetation may necessitate elevating your transmitter on a pole.

\subsection{Construction process}

Running cables to a centralised multichannel logger will often be initially the most cost efficient method, especially on sites with a large number of sensors within a small area. However, changes to the site conditions must be considered. Removing and rerouting cables can be a costly exercise and lead to gaps in data, often at critical points of the construction process. Likewise erecting structures - permanent or temporary - between a radio transmitter and its Gateway will mean a loss of communication.

\section{SI- based datalogger}

The SIM-based datalogger (Rippa) is a self-contained datalogger unit with up to two channels. The unit contains the recording hardware, long-life battery and 3G modem in an IP68 and UV stable housing. The logger will measure the instrument at a preset interval, for example, every 15 minutes and transmits all recorded data via the cellular network at a pre-set interval, typically 6-hourly. The 'store and forward' method ensures firstly data integrity; even in the event of a communications failure, all data will eventually be transmitted. It also minimises communication up time, meaning lower power consumption and lower cellular data usage. Transmitting at 24-hour intervals, the internal battery on the datalogger will support the unit for $10+$ years. At 6-hourly uploads the unit is self-sufficient for up to three years. The device uses the standards based licenced radio, allowing 2-way communication via 3G (UMTS/HSDPA) allowing data to be sent, and configuration instructions, clock synchronising and firmware updates to be received.

Data is made available through the cloud based software - web accessible software, which is used for both hardware configuration and data analysis and reporting. The software contains powerful graphing tools for live and interactive charting, as well as an export function for further processing in Excel. It allows establishment of virtual sensors for automatic compensation or comparison. It integrates Bureau of Meteorology data for barometric compensation, as well as the ability to analyse data against rainfall, temperature changes and wind speeds.

The software also contains the hardware management tools. This allows adjustment of the reading and upload interval, setting of the sensor calibration factors, establishment of virtual sensors, and displays of hardware statistics such as battery life, signal strength, last upload and next scheduled upload. Since all hardware configurations are done online no field connections are required and all commissioning can be done remotely or via the mobile specific website. The mobile software sets GPS location of the device and utilises your phone's camera for recording installation photographs. Configuration is also done on a 'store and forward' basis, compiling the logger's instructions and sending them as a packet at the next scheduled connection. For immediate configuration changes, data confirmation or commissioning, a forced connection can be made by swiping the logger with a magnet.

Using a cloud-based software offers global access, requires no IT infrastructure costs or software licensing; all software updates are done remotely and in real-time, and any firmware updates for the loggers can be 
carried out automatically. It also allows remote technical support for both data analysis tools and hardware configuration.

New low-power cellular modems, coupled with advances in long-life battery technology have allowed the practical development of self-contained cellular logging devices, with low-cost SIM card options making them extremely economical. Self-contained cellular dataloggers - simple, low-cost, internally-powered dataloggers with cellular connectivity - offer a number of advantages over short haul/unlicensed radio loggers. By utilising the cellular network rather than a local network, small projects with isolated sensors can quickly and easily establish a remote monitoring system. For example, a number of landslide projects in southeast Queensland containing only one or two piezometers can have their data telemetered and accessible by web cost effectively. For larger projects, the advantages come when sensors are likely to be relocated, or are spread over a long distance, such as highway projects or large tailings dams.

\subsection{Gateway WA project}

The Gateway WA project is a billion dollar road initiative designed to improve access to Perth Airport and surrounding industrial areas, including road and bridge improvements. The project allowed for monitoring wells spread over $6 \mathrm{~km}$ to be monitored online. When work has progressed and the boreholes are no longer of critical importance, the sensor and logger is relocated to a new position with no Gateway or network configuration required. Likewise a number of tilt sensors were used to monitor a sheet pile wall during excavation. Installation takes only as long as mounting the sensor and the logger, and commissioning only requires powering the unit up.

With regards to network stability, licensed radio - as utilised by cellular network providers - are closely monitored for spectrum crowding and quickly upgraded where spectrum crowding is a risk.

These devices do have their limitations, most obviously the requirement for and reliance upon, a cellular network. Where cellular networks are limited, short haul radio devices allow you to channel the data to a location where communications are available - external via cellular network, or internal/local via local area network (LAN), WiFi or direct connection.

\subsection{Perth City Link Project}

The Perth City Link - Rail project presented a number of challenges with regard to data delivery:

The AUD 360 million rail project, completed in 2014, is the first stage of the Perth City Link. The scope of works includes:

- Sinking the Fremantle Line between William Street and Lake/King Street. This has created almost $600 \mathrm{~m}$ of a new covered section of the Fremantle Line.

- Upgrading Perth Station with new tiling, lighting and services and convert the current Platform 9 into an island Platform 8/9.

- Created a new tunnel under the northern end of the Barrack Street Bridge to service the new Platform 9.

- Extended the existing Joondalup line tunnel roof from Lake to Milligan Streets to be in line with the new Fremantle line tunnel.

- Created a new pedestrian underpass connecting Perth Underground Station to all the platforms at Perth Station.

Existing structures were being monitored by tilt sensors and crackmeters. While the majority of these were contained within the Perth Train Station and Horseshoe Bridge (Figure 1), an area of roughly $300 \times 300 \mathrm{~m}$, many were on bridge columns in between rail tracks, or on heritage listed structures. These constraints meant running cables to central logging points was either time consuming and impractical, or outright 
impossible. This was overcome with single channel radio devices transmitting short distances to a central Gateway.

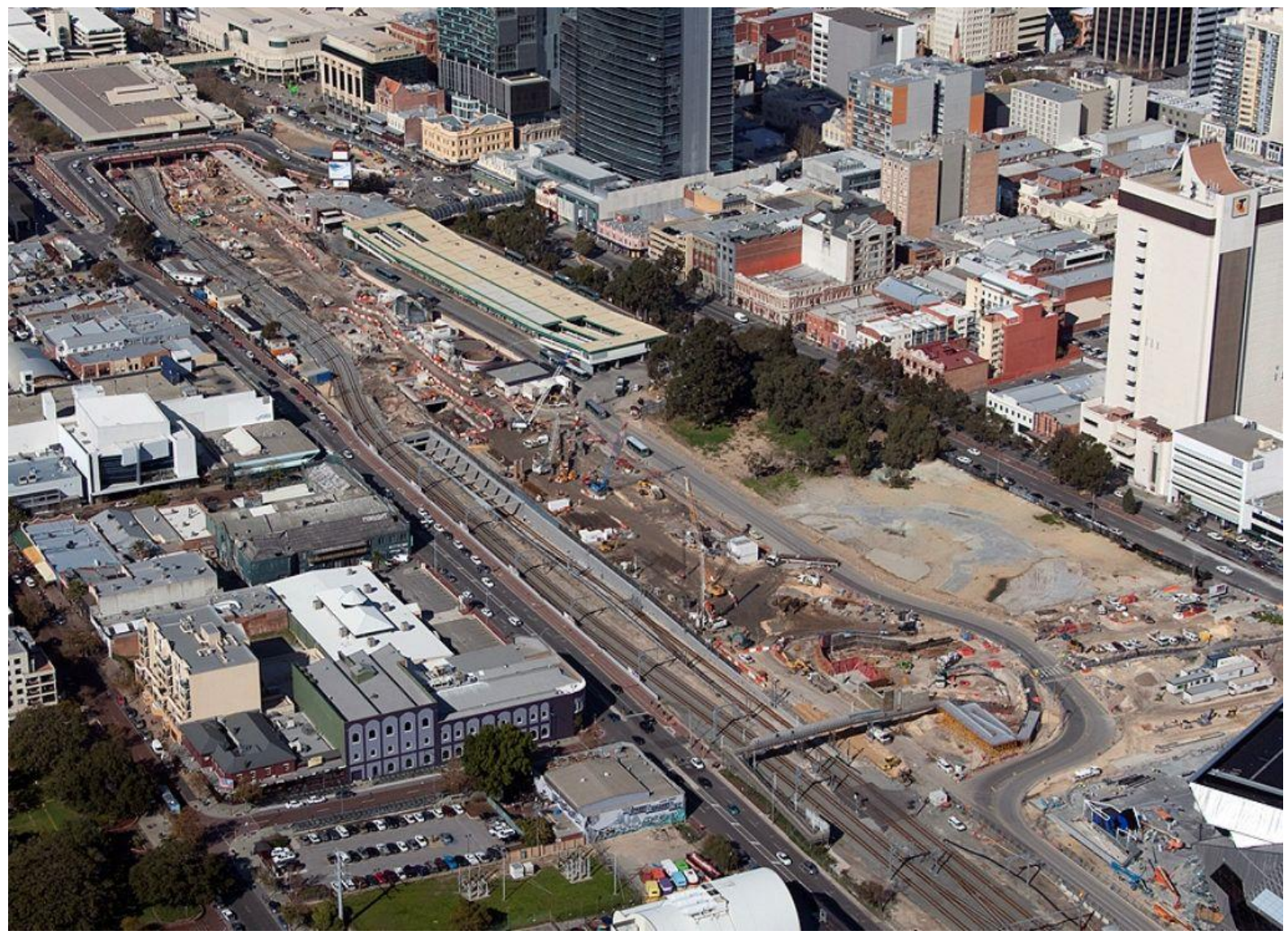

\section{Figure 1 Overview of the Perth City Link Project}

\subsection{Lowering the water table}

As the site is located above what was once Lake Kingsford, a major step in the construction process was to lower the water table, or dewater, using a number of wells installed between the tunnel walls. The water was treated to remove the high level of iron and adjust $\mathrm{pH}$ levels before being pumped back into the ground by nearby recharge wells to maintain water level around the site.

This required close monitoring of both groundwater levels and water flow from the dewatering pumps.

The purpose of the data was to ensure the water levels were matching the model with regard to the water being pumped out of the ground. This meant a clear trend plot was required, but would only be assessed once to twice per day. The sensors being monitored were pressure transducers suspended in observation wells with a 4-20 mA output, and a combination of flow meters with either a pulse output or 4-20 mA. Sensors were positioned separately and widely across the site.

We utilised the Rippa $2 \mathrm{G}$ datalogger/transmitter for recording and transmitting the data (Figure 3).

Over the life of the project approximately a gigalitre (one billion litres) of water was pumped out of the construction area, treated and pumped back into the ground. To monitor this massive undertaking the consultants opted for an innovative and successful installation of the Rippas. These units record the data and regularly upload to a dedicated web page (Figure 2) providing the engineers with near real time knowledge of how the water table is behaving. This was completely new technology and began with small numbers being installed, but as confidence grew the quantity was finally well over 100 units. 


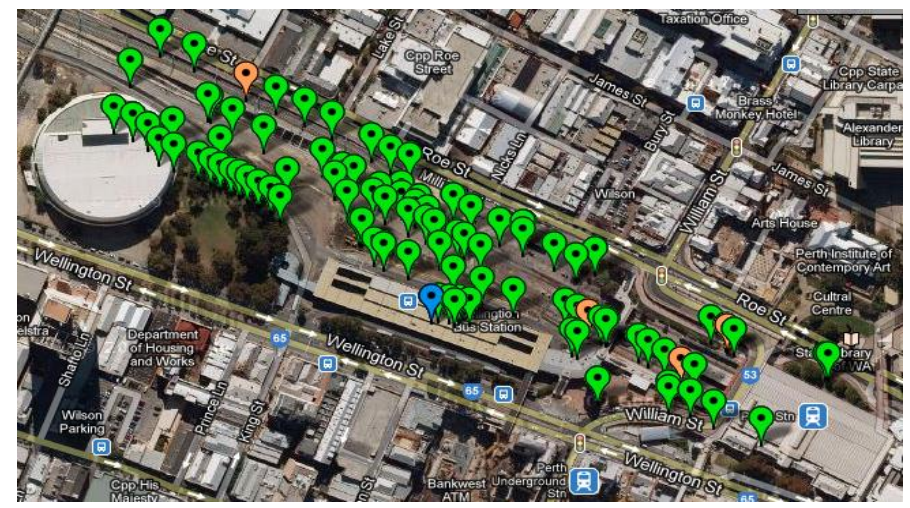

Figure 2 Software graphic of the various installations

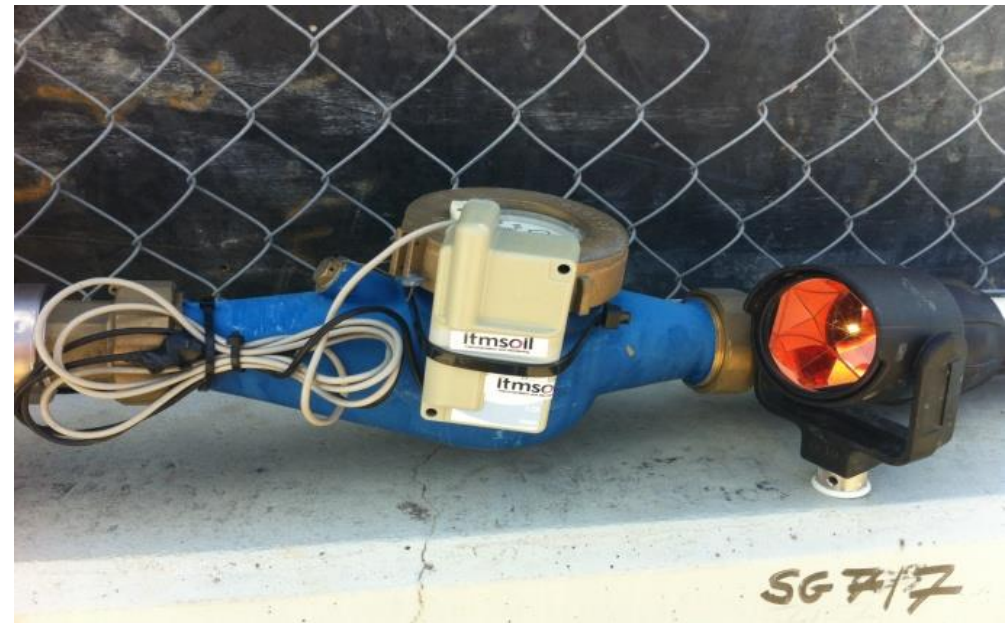

\section{Figure 3 Rippa connected to water meter}

In a site as congested as the Perth City Link Rail Alliance (PCLRA) project having cables running from the water monitoring bores to centralised dataloggers would have been impractical.

\section{Mesh networked system - technical explanation}

Wireless communications are sometimes perceived as less secure, because there is no physical connection (wires) between network devices. More specifically, there is the belief that the network can be sensitive to being hacked or provide interference problems. However, the configuration of the Loadsensing's mesh network is one of the safest solutions on the market. Loadsensing's protocol, based on frequency-hopping and time synchronisation, allows the operation of hundreds of sensors in the same network without any interference. All information broadcast by the network is encrypted, with mechanisms for verifying message integrity and authentication to access the network.

The difference and advantage facing a classical wired system is that thanks to a redundant mesh network, a malfunctioning device will be isolated, minimising the impact of his disappearance on the network, because this is dynamically adjusted to find another path to bring the data. In a classical cabling system, the break/malfunctioning of a cable most times cause a complete system loss.

The mesh technology allows each node to act as sender of its own data but also as a repeater of its neighbours (Figure 4). A network is a group of nodes with the same ID, security key, and synchronised between them. The network gateway serves as the time base for nodes and serves its configuration. The routing information is dynamic and always looking for the best path to bring data from the nodes to the gateway. 


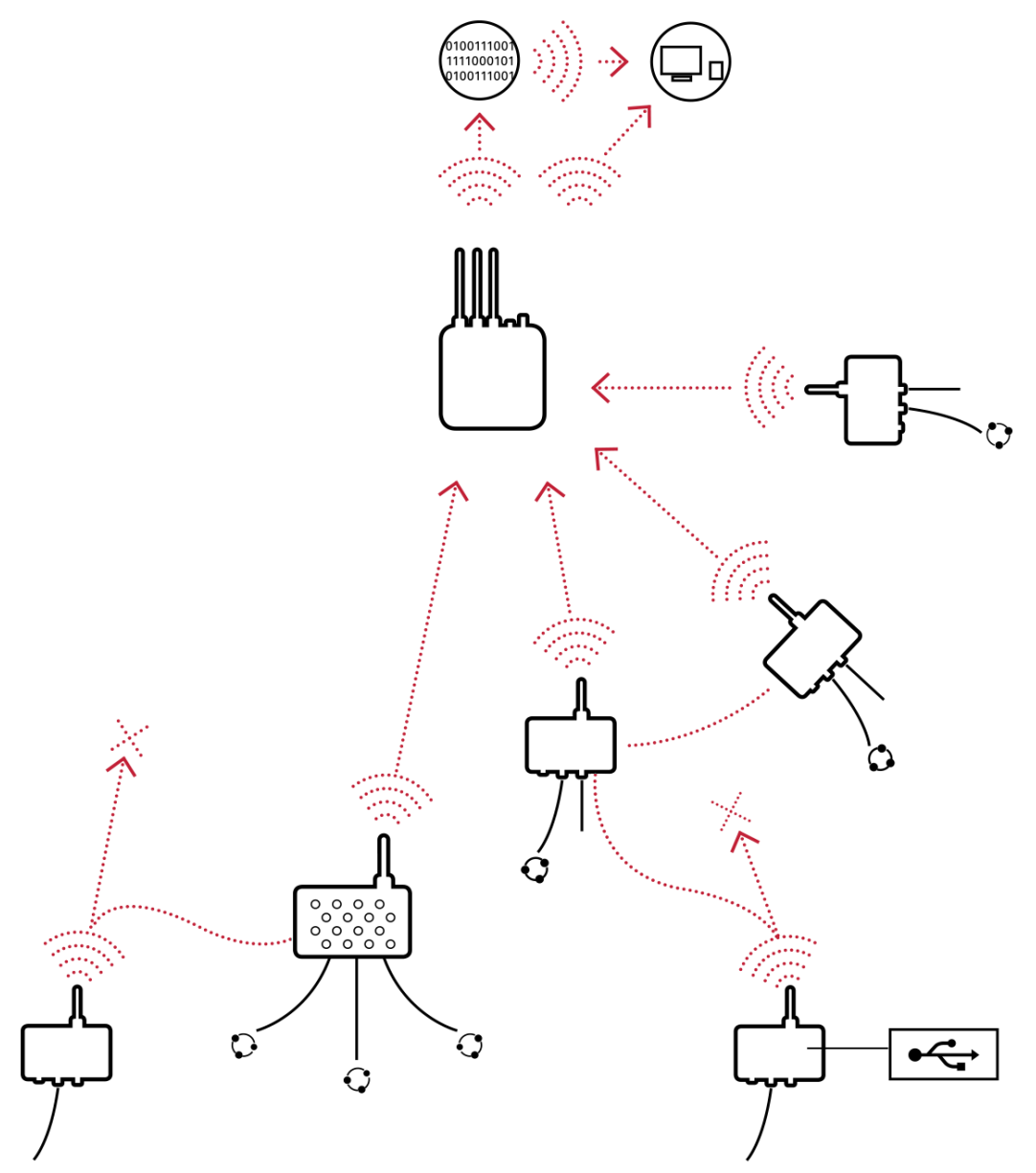

Figure 4 Diagram of a load sensing (LS) wireless network

When a new node joins the network, it is assigned an ID and receives information from time and frequency synchronisation of its neighbours. This ensures that the network is working properly synchronised with the new device. This allows the operation of different networks in one place without interaction between them.

The wireless nodes are activated periodically, following its programming. Additionally, to improve the robustness of communications different communication channels are used that exist at $2.4 \mathrm{GHz}$ frequency band, using a technique called channel-hoping which avoids interference: each data packet sent from one node to its neighbour is sent by a different channel, choosing the best available channel depending on the level of interference observed in the different channels.

All devices in a LS network share the same time base; this means that each node transmits, hears or sleeps during time slots assigned. Each time slot has a length of a few milliseconds, this keeps the devices asleep most of the time and only be active in the sending window data. This allows you to create not only highly secure and robust networks, but also a very low power performance that allows years of autonomy running on standard internal batteries.

The wireless communication is based on long used industrial standards known as highway addressable remote transducer (HART). The HART standard is one of the first implementations of the standard Fieldbus also well known in industrial environments. The current specification on which the communication system is based is HART 7 September 2007: in this specification an included section is defined as Wireless HART, which has been implemented in the LS nodes. The protocol offers a fully synchronous communication Mesh type dynamic network: this ensures redundancy in communications, resilience and resistance to failures, and thus ensures the reliability of the communication system even in the most complex environments. 
Additionally, the network synchronisation allows:

- In each window, submission seeks the sending node that is best to send the data packet.

- In case of specific high demand network reset communication times for all data packets to be delivered on time.

- The possibility of negotiating the frequency in each window of communication, offering a highly robust system against radio frequency interference. The system used is called frequency hopping spread spectrum (FHSS) combined with a method called direct sequence spread spectrum (DSSS), offering significant advantages in terms of scope and protection against fading.

Furthermore, we implement the following security systems:

- Confidentiality: there is an encryption system data using the algorithm (Hardware) 128-bit AES. This prevents interception of packets.

- Data integrity: each message sent is protected by a code that looks the integrity packet sent. If this is not correct the packet is forwarded.

- Protection against replay: replay attacks are protected by individual meters on each node to ensure its invulnerability network level.

- Access control: each packet sent by a node carries an ID generated by the node itself. On receiving each packet is checked with a unique key of the Gateway.

- Compartmentalised security: the loss of a portion of a data packet does not compromise the network. Part of lost data is recovered in successive retransmissions.

- Mechanically the LS nodes are also very robust. These are protected with an IP67 encapsulated to protect them from rain, shock, lightning, dust etc.

\section{$4 \quad$ Case studies}

\subsection{Slope stability}

Monitoring is fundamental for the prediction and analysis of landslide triggering factors and dynamic behaviour, major issues in the hazard assessment and risk mitigation. Wired monitoring systems have traditionally been used in landslide monitoring. However, wireless technologies are escalating in this field as a consequence of their multiple advantages against standard wired systems, such as their versatility or their lower power consumption. Wireless monitoring is the perfect solution for the acquisition of data on geological processes placed in remote areas where power availability is scarce, and the position of the sensors is often a critical issue due to the landscape conditions. In this context, a complete landslide wireless monitoring system was successfully installed in the Rebaixader catchment (Central Pyrenees, Spain) (Hürlimann et al. 2014).

The Rebaixader constitutes a typical high mountain catchment where landslides and torrential processes occur with a sub-annual frequency. Rainfall is the principal triggering factor of the type of landslides occurring at the Rebaixader known as debris flows, but the specific details of the geotechnical mechanisms that originate the events are still not clear. In order to increase the knowledge on the processes occurring at the catchment, this was equipped with a LS network for the monitoring of triggering factors and a Worldsensing's Spidernano Seismic Remote Unit for the acquisition of the ground vibration generated by the moving mass (Figure 5).

The sensor network includes seven low-power wireless dataloggers. Digital, pulse and voltage sensors are connected to these dataloggers in order to monitor soil water content, soil water potential, snow height, 5-minute rainfall intensity and air temperature and humidity. The dataloggers communicate in a multi-hop fashion to deliver the information into the gateway, placed $1 \mathrm{~km}$ far down the hill but in line-of-sight, with 
two repeaters placed along the line. The gateway offers enhanced computational and storage capabilities as well as $3 \mathrm{G}$ modem communication to the data centre.

The Spidernano seismic datalogger was connected to three 1D geophones recording simultaneously the ground vibration generated by the pass of the debris-flow mass. The Seismic Remote Unit (SRU) equipped with GPS clock discipline, and is placed nearby the gateway and connected to it via Ethernet. The SRU has a low power consumption $(0.5 \mathrm{~W})$, specially adapted for field campaigns or permanent monitoring.

The recordings of SRU revealed that debris-flow occurrence can be perfectly detected by ground vibration signal, and the approach of the flowing mass can even be detected before the arrival. Data acquired by the wireless sensor network provided valuable results on the understanding of the failure and post-failure mechanisms such as the $15 \mathrm{~mm}$ in one hour as a preliminary rainfall threshold for debris-flow initiation in summer season. All these achievements are promising results for the application of low power wireless technologies not only for standard landslide monitoring but also for landslide early warning systems.

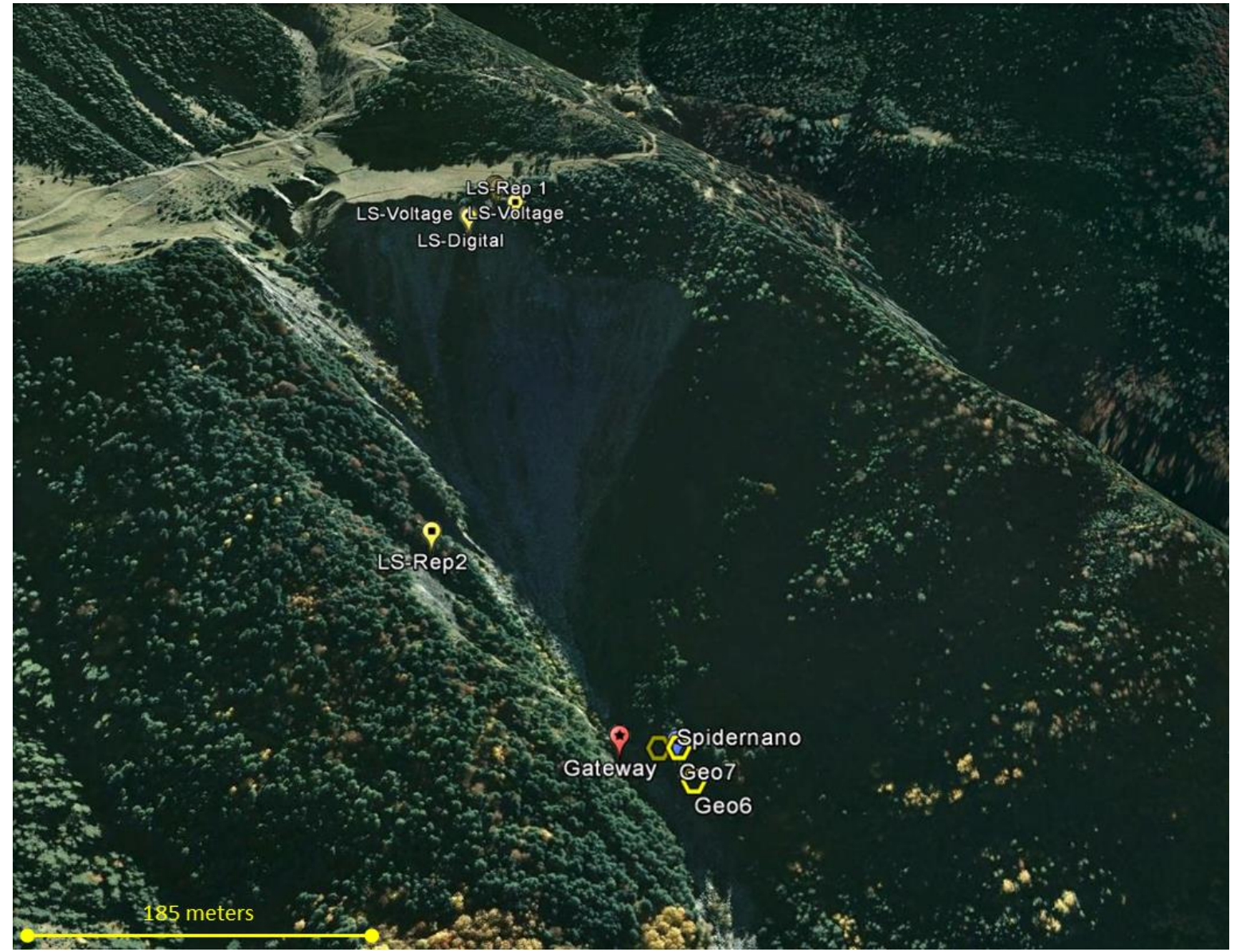

Figure 5 Rebaixader catchment, Central Pyrenees (Catalonia)

Figure 5 shows LS wireless dataloggers are placed on top of the catchment to monitor triggering conditions, one seismic datalogger is located at the base of the catchment to monitor ground vibration after debris flow and mass movement. One repeater was located at the middle of the catchment to repeat data from the dataloggers to reach the gateway, which gathers data from LS dataloggers and the seismic unit.

\subsection{Rockfall}

Railways crossing mountainous regions are often threatened by rockfalls. On one side, trains circulating along threatened areas are exposed to relevant hazards. On the other side, damages caused by rockfalls produce delays in railway traffic due to unexpected events, which usually generates important economic losses. Supervision of these areas results in costly operations of maintenance for the railway operators. 
Aiming to provide an optimal solution to improve operations for ÖBB (Austrian Railways) two solutions using LS wireless sensor networks have been implemented.

First, an automatic low-power warning system implementing wireless dataloggers is being used in the railway crossing the Hieflau tunnel, in central Austria. An unstable rock mass, in the middle of a rock wall at the tunnel entry, is directly threatening the railway (Figure 6). Two crackmeters connected to a datalogger have been installed to monitor a critical rock aperture of the unstable mass, in the middle of the rock wall. The crack aperture is being monitored every hour and data is processed in order to determine whether it exceeds a predefined threshold. In case that the aperture threshold is exceeded, an e-mail is sent to ÖBB. Thanks to the LS wireless system the occurrence of rockfall events can be predicted at a real low cost of installation and maintenance. Thanks to this information, authorities are able to ask maintenance operators to be there only when necessary (LS alarm notification) and without any need of long cable revisions, avoiding periodical expensive on-site operations.

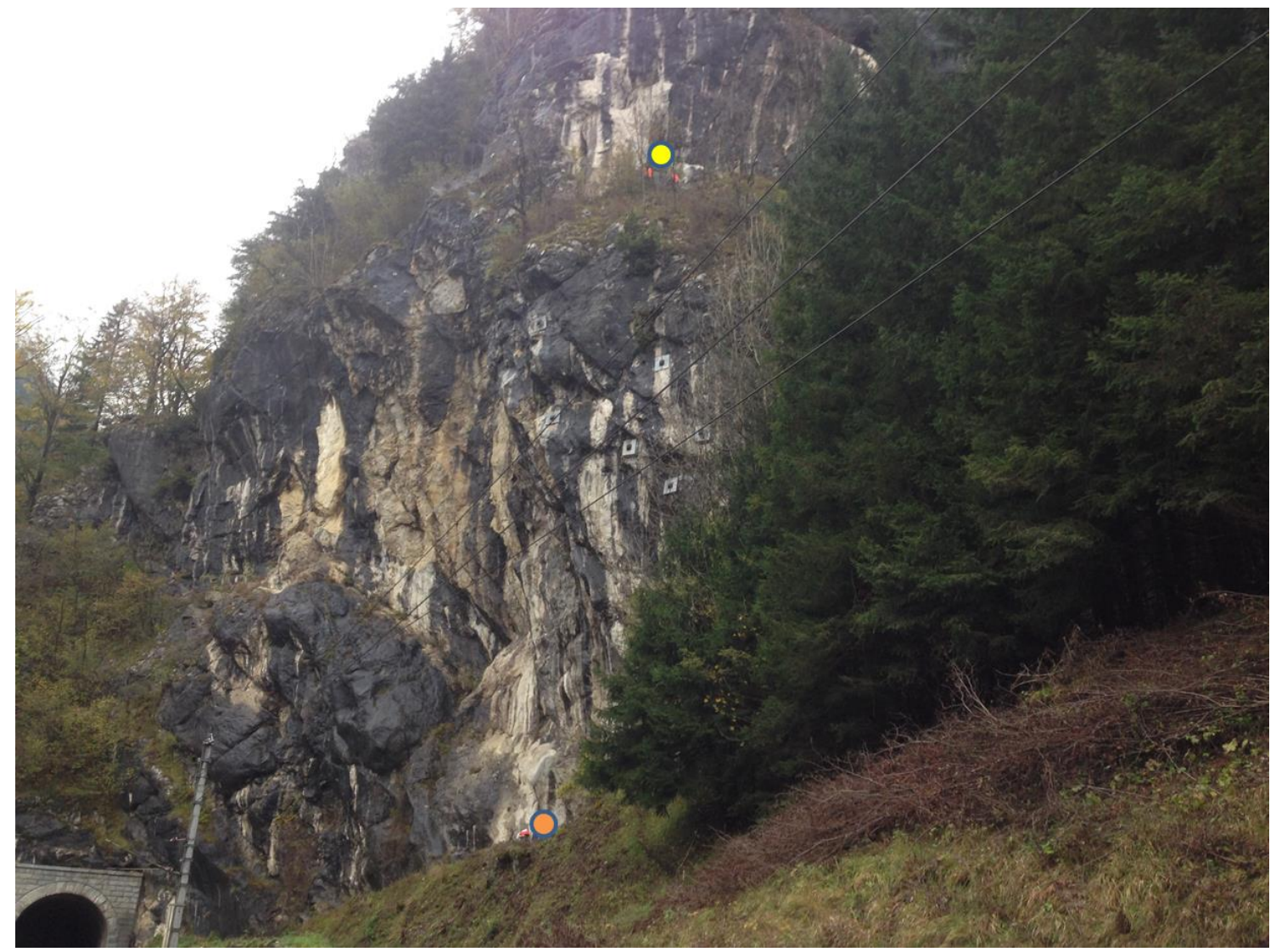

\section{Figure 6 Hieflau tunnel, central Austria}

Second, the LS system has been implemented to monitor rockfall barriers to prevent rockfall events in Austrian railways. This project is based on multiple sensors, which are combined to form a wireless radio network of the nets. Strain gauge load cells measure the tension of the steel cable of the barrier, while tiltmeters (+-180 range) measure the barrier inclination, and wire extensometers measure the barrier movement (Figure 7). Each type of sensor is read by a wireless datalogger, which at the same time creates a wireless network that sends all data to the gateway. The gateway (solar-powered) delivers all data via global system for mobile communication to the customer's servers. This low-maintenance and self-sufficient solution provides a 24 hour monitoring of the rockfall barriers in inaccessible areas in all weather conditions, delivering data every hour, and in case of particular events, the sampling rate can be increased remotely thanks to the bi-directional capacities of the system. Thanks to this, the rockfall barriers can be monitored in real time without any need for human maintenance in a difficult-to-access remote site. 


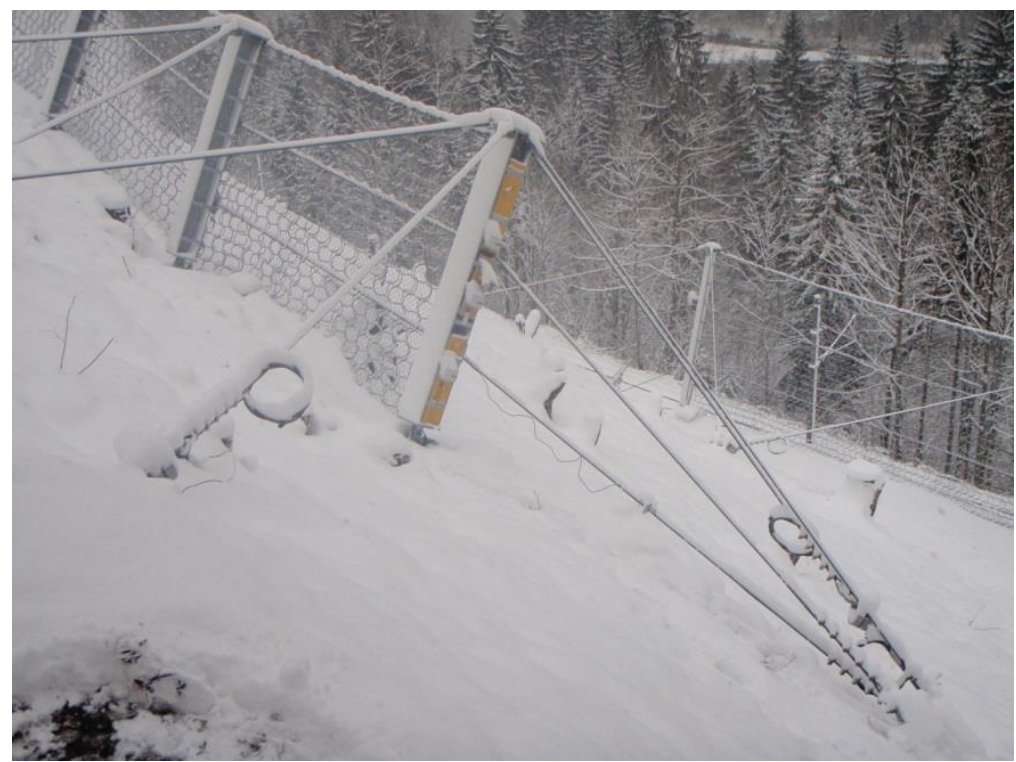

Figure 7 Rockfall barrier, central Austria

\subsection{Roy Hill Project}

Roy Hill is an iron ore mining project in the Chichester Range in the Pilbara region of Western Australia, located 115 kilometres $(71 \mathrm{mi})$ north of Newman and 277 kilometres $(172 \mathrm{mi})$ south of Port Hedland.

The project requires a new railway from the mine to port to move the ore to market in the quickest time possible. A major section of this construction is the rail loop at the port which required a significant embankment to be constructed on soft marine material. Standard instrumentation would typically be settlement cells. In an effort to expedite construction the contractor wanted to automate the readings and remove the settlement plates from being an obstruction to the truck movements. To achieve this requirement settlement cells were installed. This then raised the next issue of collecting the data in the most appropriate method. Many scenarios were contemplated until it was decided to use the mesh network wireless system.

With an active construction site approximately 1,500 by $1,000 \mathrm{~m}$ incorporating 45 settlement cells and 14 piezometers it was a problematic installation. The wireless system relayed all the data to three Gateways (Figures 8 and 9), incorporating a SIM card that transmitted the results to LS's internet based software for easy access and alarms (Figure 8 ).

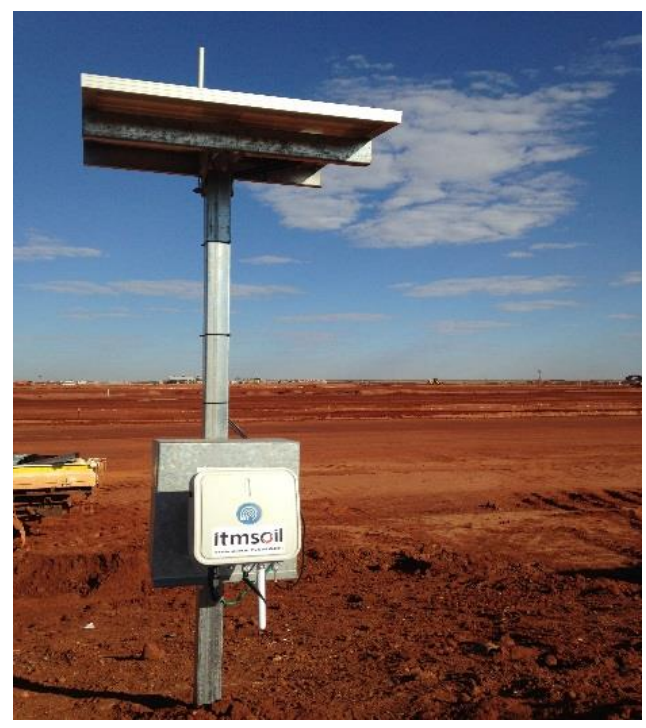

Figure 8 Gateway installations 


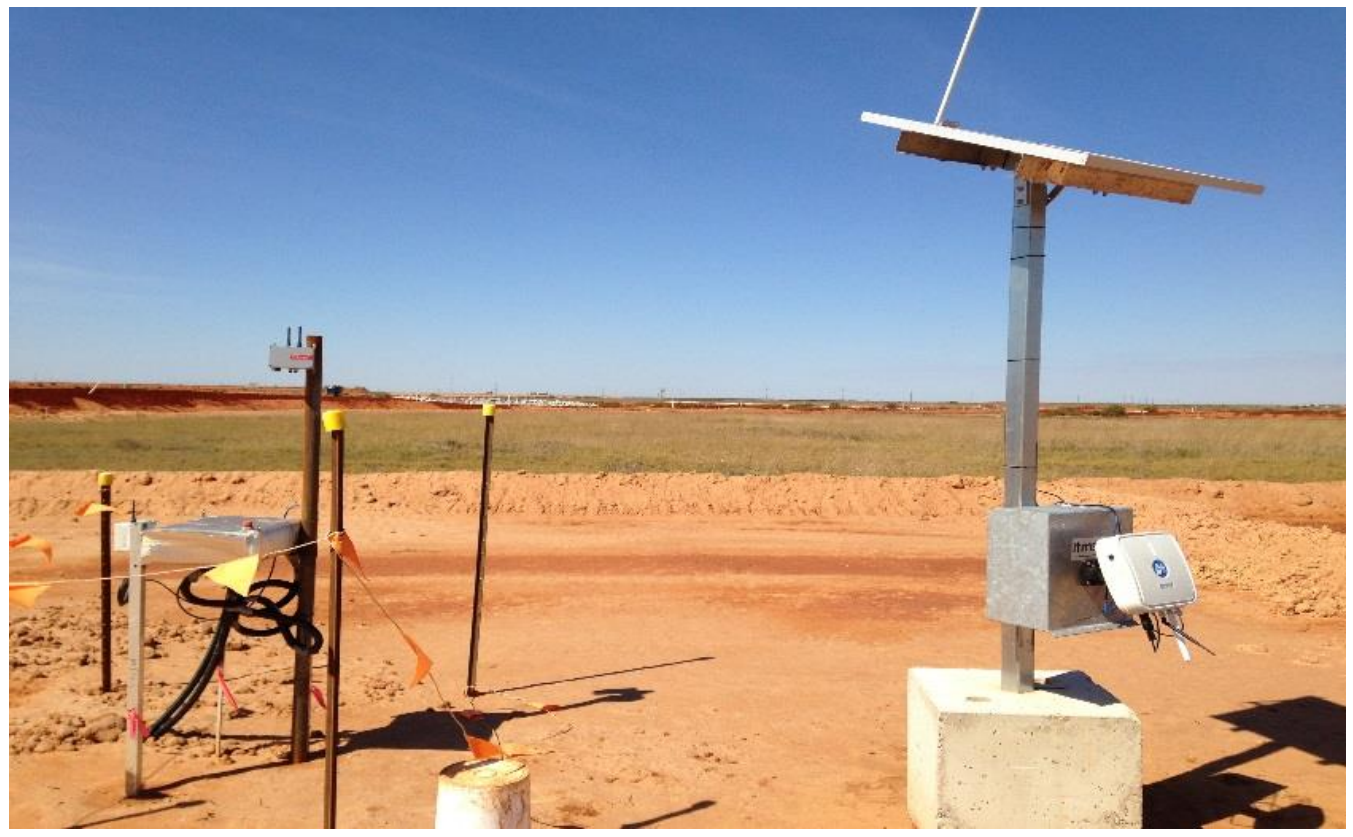

\section{Figure 9 Second Gateway with node in close proximity}

\section{$5 \quad$ Future}

Wireless sensor networks (WSN) have mostly been based on the concept of tiny devices, with a set of sensors and a radio to send the results to a dedicated server or the Internet. This radio infrastructure has been typically designed keeping in mind the state-of-the-art on radio transceivers with low power consumption, but implying a short range (100 $\mathrm{m}$ max). The solution to large projects that almost everybody has found has led to develop and build a mesh network of devices, with a medium to low range between the nodes of the mesh. In these designs, data is sent from one device to another until the data reaches the central node (gateway) which itself is connected to the Internet in a more traditional way (3G, GPRS, Ethernet).

However, depending on the applications, mesh networks might present several problems. One of them is the synchronisation between devices; because each device has a different clock drift, it is often mandatory to synchronise the whole network, leading to raising energy consumption. Another problem is the asymmetry on the devices consumption, meaning that not all devices drain power at same rate. This is because nodes closer to the gateway will act as repeaters to other devices. This phenomenon complicates the operational costs of the network maintenance, even limiting the use of energy harvesting techniques in some of the devices in the same network. Other operational costs of mesh networks are in the use of repeaters to enhance coverage of some parts of the deployment, and many times it is not easy to detect these dark zones. The last problem arises on networks where the density of nodes is not high (due to costs of the nodes depending on the application). In these kinds of networks, the entire network sometimes relies on a single node to repeat the signal form others, and the malfunctioning of this node can break down the communication of all data.

Recently, a new concept of radio systems has arisen. This new idea is focused on sending few bits of data (enough for the sampling rates and data used in geotechnical instrumentation) at a very slow transmission rate (less than $1 \mathrm{kbps}$ ) but with a long range (up to kilometres). These solutions are based on techniques like spread spectrum and specifically designed modulation schemes for high sensitivity. With this new concept of radio in mind, the landscape of WSNs is changing. The use of a mesh network is no longer needed, because with these long-range radios all devices can communicate directly with the gateway. Hence, from a mesh network topology we are changing to a star network, and a single gateway receives data directly from all devices. This implies that the loss of one device does not affect any other device. 
This change simplifies enormous operational procedures and costs, because it avoids the use of repeaters. It also simplifies coverage tests and, hence, usually needs fewer trained people to do the installation. Once these new long-range radios are implemented in the geotechnical/structural monitoring world, deploying a WSN will be as easy as placing the sensor+datalogger wherever fits best, checking that they are covering your deployment zone and just dropping your devices and starting to receive data into your servers. There is no need of complex planning to deploy a mesh network. That is, concentrating on what your true business is: providing services to specific industries (and not spending most of your efforts in dealing with coverage problems).

\section{Reference}

Hürlimann, M, Abancó, C, Moya, J \& Vilajosana, I 2014, 'Results and experiences gathered at the Rebaixader debris-flow monitoring site, Central Pyrenees, Spain', Landslides, vol. 11, no. 6, pp. 939-953. 